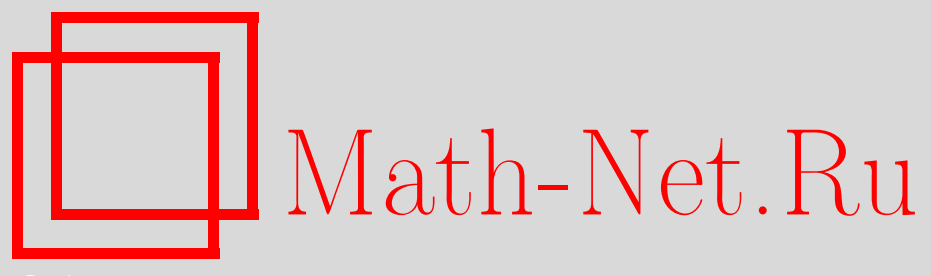

С. А. Кордюкова, Иерархия Кортевега-де Фриза как асимптотический предел системы Буссинеска, ТMФ, 2008, том 154, номер 2, 294-304

DOI: https://doi.org/10.4213/tmf6170

Использование Общероссийского математического портала Math-Net.Ru подразумевает, что вы прочитали и согласны с пользовательским соглашением http://www . mathnet.ru/rus/agreement

Параметры загрузки:

IP : 34.227 .88 .159

26 апреля 2023 г., 14:48:07

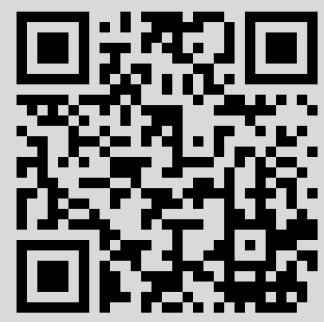




\title{
2008 г.
}

\section{С. А. Кордюкова*}

\section{ИЕРАРХИЯ КОРТЕВЕГА-ДЕ ФРИЗА КАК АСИМПТОТИЧЕСКИЙ ПРЕДЕЛ СИСТЕМЫ БУССИНЕСКА}

\begin{abstract}
Для модели поверхностных волн проводится асимптотический анализ по малому параметру $\varepsilon$ на далеких временах, где требуется учитывать поправки к приближению, описываемому уравнением Кортевега-де Фриза. Обнаружено появление иерархии Кортевега-де Фриза, которая обеспечивает построение асимптотики вплоть до времен $t \approx \varepsilon^{-2}$, где приближение Кортевега-де Фриза становится непригодным.
\end{abstract}

Ключевые слова: нелинейное уравнение, малый параметр, потенцированное уравнение Кортевега-де Фриза, канонический оператор Ли-Беклунда, метод многих масштабов, асимптотика, солитон.

\section{1. ВВЕДЕНИЕ}

Асимптотические методы дают эффективные способы анализа сложных нелинейных систем, в частности моделей поверхностных волн. Применение этих методов основано на наличии малого параметра в исходных уравнениях. В модели длинных волн на мелкой воде в качестве малого параметра $0<\varepsilon \ll 1$ выступает квадрат отношения вертикального масштаба к горизонтальному [1]. В таком случае исходные уравнения для плоского одномерного движения жидкости записываются в виде

$$
\begin{aligned}
\phi_{x x}+\varepsilon^{-1} \phi_{y y} & =0, & & x \in \mathbb{R}, \quad 0<y<h(x, t) ; \\
h_{t}+h_{x} \phi_{x}-\varepsilon^{-1} \phi_{y} & =0 & & \text { при } \quad y=h(t, x) ; \\
\phi_{t}+\frac{1}{2} \phi_{x}^{2}+\frac{1}{2} \varepsilon^{-1} \phi_{y}^{2}+g h & =0 & & \text { при } \quad y=h(t, x) .
\end{aligned}
$$

Здесь $\phi(x, y, t)$ - потенциал скорости, свободная поверхность задается уравнением $y=h(x, t)$. Глубина невозмущенной жидкости $h_{0}=$ const. Уравнения дополняются условием непротекания $\phi_{y}=0$ на горизонтальном дне при $y=0$. Для степеней производных всюду ниже используются обозначения типа $\phi_{x}^{2}=\left(\phi_{x}\right)^{2}, \phi_{x x}^{2}=\left(\phi_{x x}\right)^{2}$ и т.д.

*Уфимский государственный авиационный технический университет, Уфа, Россия. E-mail: sveta.kor05@mail.ru 
Идеи асимптотического анализа позволяют упростить исходные сложные уравнения до приемлемого уровня. Известно, что в нулевом (главном) порядке по $\varepsilon$ система (1)-(3) сводится к волновому уравнению, решение которого дает пару простых плоских волн. Такое приближение пригодно на не слишком больших временах: $|t| \ll \varepsilon^{-1}$. В более точной модели возникает уравнение Буссинеска четвертого порядка [1], [2]. Формально оно представляет собой возмущение волнового уравнения. Уравнение Буссинеска асимптотически можно редуцировать к паре уравнений Кортевега-де Фриза (КдФ), решения которых описывают медленную деформацию плоских волн в масштабе $\tau_{1}=\varepsilon t$. Такие приближения пригодны на довольно больших временах: $|t| \ll \varepsilon^{-2}[1]-[3]$.

Чтобы получить приближение, пригодное на следующем масштабе времени $\tau_{2}=$ $\varepsilon^{2} t$, необходимо учитывать поправки более высокого порядка в уравнении Буссинеска. Именно эта проблема рассматривается в данной работе. Похожие задачи неоднократно исследовались в теории возмущения солитонов [4]. Однако возмущения, которые возникают из уравнений поверхностных волн в случае ровного дна, оказываются весьма специфическими. Эта специфика приводит к тому, что возмущение солитона КдФ проявляется лишь в медленном, линейном по времени сдвиге фазы без изменения амплитуды. Фактически в этом состоит один из результатов данной работы. Но наиболее важным результатом является получение для главных членов асимптотики системы уравнений, состоящей из уравнений КдФ-иерархии (потенцированных).

Асимптотические решения уравнения Буссинеска, полученные в этой работе, основаны на преобразованиях Ли-Беклунда потенцированного уравнения КдФ. Такой подход отличается от известных подходов в теории возмущения солитонов [4] и представляется перспективным для анализа решений, отличных от односолитонных. Данная работа является развитием идей, изложенных в работах [5].

\section{2. РЕДУКЦИЯ СИСТЕМЫ ПОВЕРХНОСТНЫХ ВОЛН К УРАВНЕНИЮ БУССИНЕСКА}

Первый этап построения асимптотического решения состоит в переходе от задачи (1)-(3) с двумя пространственными переменными $(x, y)$ к уравнению с одной пространственной переменной $x$. С этой целью для потенциала как решения уравнения (1) с малым параметром $\varepsilon$ используется асимптотическое представление через новую неизвестную функцию двух переменных $A(x, t, \varepsilon)$ (см. [1]):

$$
\phi(x, y, t, \varepsilon)=A(x, t, \varepsilon)+\sum_{n=1}^{\infty}(-1)^{n} \varepsilon^{n} \frac{y^{2 n}}{(2 n) !} \partial_{x}^{2 n} A, \quad x \in \mathbb{R}, \quad 0 \leqslant y \leqslant h, \quad \varepsilon \rightarrow 0
$$

В таком случае уравнения на поверхности (2), (3) можно записать в асимптотической форме с любым порядком точности (по $\varepsilon)$. Используя разложение (4) до порядка $\varepsilon^{2}$, 
преобразуем уравнения (2), (3) к виду

$$
\begin{aligned}
h_{t}+ & h_{x} A_{x}+A_{x x} h-\frac{\varepsilon}{2} h^{2}\left(A_{x x x} h_{x}+\frac{1}{3} A_{x x x x} h\right)+ \\
& +\frac{\varepsilon^{2}}{4 !} h^{4}\left(A_{x x x x x} h_{x}+\frac{1}{5} A_{x x x x x x} h\right)=O\left(\varepsilon^{3}\right), \\
A_{t}+ & \frac{1}{2} A_{x}^{2}+g h-\frac{\varepsilon}{2} h^{2}\left(A_{x x t}+A_{x} A_{x x x}-A_{x x}^{2}\right)+ \\
& +\varepsilon^{2} h^{4}\left(\frac{1}{4 !} A_{x x x x t}+\frac{1}{8} A_{x x x}^{2}-\frac{1}{6} A_{x x} A_{x x x x}\right)=O\left(\varepsilon^{3}\right), \quad \varepsilon \rightarrow 0 .
\end{aligned}
$$

Заметим, что функцию $A(x, t, \varepsilon)$ можно связать со значением потенциала $\phi$ на поверхности $y=h$, обратив асимптотически равенство (4):

$$
A(x, t, \varepsilon)=\left[\phi+\frac{\varepsilon}{2} h^{2} \phi_{x x}+\frac{5 \varepsilon^{2}}{4 !} h^{4} \phi_{x x x x}+\cdots\right]_{y=h(x, t, \varepsilon)} .
$$

Помимо того, для дальнейшего анализа удобно выделить главные члены асимптотики, используя формулы

$$
h=h_{0}+\varepsilon \eta(x, t, \varepsilon), \quad \phi=-g h_{0} t+\varepsilon \psi(x, t, \varepsilon) \quad \text { при } \quad y=h(x, t, \varepsilon) .
$$

Если подставить (6), (7) в соотношения (5), то для функций $\eta, \psi(x, t, \varepsilon)$ получается система уравнений в асимптотической форме, которая в главных порядках имеет вид

$$
\begin{aligned}
& \eta_{t}+h_{0} \psi_{x x}+ \varepsilon\left(\left(\psi_{x} \eta\right)_{x}+\frac{1}{3} h_{0}^{3} \psi_{x x x x}\right)+\varepsilon^{2}\left(h_{0}^{2}\left(\psi_{x x} \eta\right)_{x x}+\frac{16}{5 !} h_{0}^{5} \psi_{x x x x x x}\right)=O\left(\varepsilon^{3}\right), \\
& \psi_{t}+g \eta+\frac{\varepsilon}{2} \psi_{x}^{2}+\varepsilon^{2}\left(h_{0} \eta_{t} \psi_{x x}+\frac{h_{0}^{2}}{2} \psi_{x x}^{2}\right)=O\left(\varepsilon^{3}\right), \quad \varepsilon \rightarrow 0
\end{aligned}
$$

Полученные уравнения (8), (9) принято называть системой Буссинеска [1], [2]. Их можно преобразовать к одному уравнению с точностью до $O\left(\varepsilon^{3}\right)$. Для этого достаточно выразить $\eta$ из уравнения (9) и подставить в уравнение (8), при этом получим следующее уравнение Буссинеска:

$$
\begin{aligned}
& -\frac{1}{g} \psi_{t t}+h_{0} \psi_{x x}+\frac{\varepsilon}{g}\left(\frac{1}{3} h_{0}^{3} g \psi_{x x x x}-2 \psi_{x} \psi_{t x}-\psi_{t} \psi_{x x}\right)+ \\
& \quad+\varepsilon^{2}\left(\frac{-h_{0}^{2}}{g}\left(\psi_{40} \psi_{t}+2 \psi_{x x x} \psi_{t x}+2 \psi_{x x} \psi_{t x x}\right)-\right. \\
& \left.\quad-\frac{3}{2 g} \psi_{x x} \psi_{x}^{2}+\frac{h_{0}}{g^{2}}\left(\psi_{t t} \psi_{x x}\right)_{t}+\frac{2}{15} h_{0}^{5} \psi_{x x x x x x}\right)=O\left(\varepsilon^{3}\right), \quad \varepsilon \rightarrow 0
\end{aligned}
$$

Далее делается преобразование с целью исключения лишних констант из уравнения (10): $x=z \sqrt{h_{0} g}, t=t, \psi=-h_{0}^{2} u / 9, \epsilon=2 \varepsilon h_{0} /(3 g)$. Таким образом, остается лишь один параметр $\epsilon$, который считается малым: $0<\epsilon \ll 1$, и уравнение $(10)$ 
приводится к виду

$$
\begin{aligned}
u_{t t}- & u_{z z}-\frac{\epsilon}{2}\left(u_{z z z z}+\frac{2}{3} u_{z} u_{t z}+\frac{1}{3} u_{t} u_{z z}\right)+\epsilon^{2}\left(-\left(u_{t} u_{z z}\right)_{z z}-u_{z z} u_{t z z}+\right. \\
& \left.+\frac{1}{6} u_{z z} u_{z}^{2}+\left(u_{z z} u_{t t}\right)_{t}-\frac{6}{5} u_{z z z z z z}\right)=O\left(\epsilon^{3}\right), \quad \epsilon \rightarrow 0 .
\end{aligned}
$$

Уравнение Буссинеска с точностью до второго порядка по $\epsilon$ рассматривалось в работе [6], где было получено нелокальное (псевдодифференциальное) уравнение с квадратичной нелинейностью, учитывающее все члены дисперсии и влияние медленно меняющегося дна.

\section{3. СИСТЕМА ПОТЕНЦИРОВАННЫХ УРАВНЕНИЙ КДФ-3 И КДФ-5: РЕШЕНИЯ С ПОСТОЯННОЙ АМПЛИТУДОЙ}

В этом разделе указывается класс решений системы уравнений, состоящей из потенцированных уравнений КдФ-3 и КдФ-5, при помощи которых строится асимптотическое решение уравнения типа Буссинеска.

Рассмотрим систему первых двух уравнений иерархии потенцированных уравнений КдФ:

$$
\begin{aligned}
& a_{\tau}=\frac{1}{2} a_{x}^{2}+a_{x x x}, \\
& a_{T}=\left(\frac{1}{6} a_{x}^{3}+a_{x} a_{x x x}+\frac{1}{2} a_{x x}^{2}+\frac{3}{5} a_{x x x x x}\right) c,
\end{aligned}
$$

где $a=a(x, \tau, T)$ - искомая функция, а $c$ - произвольная постоянная. Для уравнения КдФ-3 хорошо известно односолитонное решение $u=2 k^{2} / \operatorname{ch}^{2}\left(k\left(x-v t+x_{0}\right)\right)$, где $v=4 k^{2}$, при любых $k, x_{0}=$ const. Аналог односолитонного решения для системы (12), (13) дается выражением

$$
a(x, \tau, T)=2 k \operatorname{th}\left(k\left(c_{1} x+c_{2} k^{2} \tau+c_{3} k^{4} T c+c_{0}\right)\right),
$$

где $k, c_{0}$ - произвольные постоянные. При подстановке решения (14) в систему (12), (13) постоянные $c_{1}, c_{2}, c_{3}$ вычисляются точно: $c_{1}=1 / 6, c_{2}=1 / 54, c_{3}=$ $1 / 810$.

Более того, в подобной форме можно записать точное решение первых $p$ уравнений КдФ-иерархии:

$$
a\left(x, T_{1}, \ldots, T_{p}\right)=2 k \operatorname{th}\left(k\left(c_{1} x+\sum_{i=1}^{p} c_{i+1} T_{i} k^{2 i}\right)+c_{0}\right)
$$

при любых $k, c_{0}=$ const. В данной работе используется решение (14) наиболее простого вида с $c_{0}=0$.

\section{4. ПРИМЕНЕНИЕ ПРЕОБРАЗОВАНИЙ ЛИ-БЕКЛУНДА ДЛЯ РЕШЕНИЯ ЛИНЕАРИЗОВАННЫХ УРАВНЕНИЙ}

Здесь мы приводим один из способов решения линеаризованных уравнений, которые соответствуют нелинейным уравнениям в частных производных. 
Рассмотрим уравнение в частных производных с параметром $\varepsilon$

$$
F^{0}\left(t, x, u_{t}, u, u_{x}, u_{x x}, \ldots\right)+\varepsilon F^{1}\left(t, x, u_{t}, u, u_{x}, u_{x x}, \ldots\right)=0 .
$$

Пусть $u^{0}=u^{0}(x, t)$ - точное решение уравнения

$$
F^{0}\left(t, x, u_{t}, u, u_{x}, u_{x x}, \ldots\right)=0 .
$$

Если функция

$$
u=u^{0}(x, t)+\varepsilon u^{1}(x, t)
$$

является формальным асимптотическим решением уравнения (15) с невязкой $O\left(\varepsilon^{2}\right)$, $\varepsilon \rightarrow 0$, то $u^{1}$ удовлетворяет линейному уравнению, полученному в первом порядке по $\varepsilon$ при подстановке (17) в (15). Такое уравнение будем называть линеаризацией уравнения (16) на решении $u^{0}$ или линеаризованным уравнением.

Напомним, что каноническим оператором Ли-Беклунда называется оператор вида

$$
X_{\eta}=\eta \frac{\partial}{\partial u}+\left(D_{x} \eta\right) \frac{\partial}{\partial u_{x}}+\left(D_{t} \eta\right) \frac{\partial}{\partial u_{t}}+\left(D_{x x} \eta\right) \frac{\partial}{\partial u_{x x}}+\cdots
$$

где $\eta=\eta\left(t, x, u, u_{x}, u_{x x}, \ldots\right) ; D_{x}$ и $D_{t}$ - операторы полной производной, $D_{x x}=D_{x}^{2}=$ $D_{x}\left(D_{x}\right), D_{x x x}=D_{x}^{3}=D_{x}\left(D_{x x}\right)$ и т.д.

Лемма. Пусть существует канонический оператор Ли-Беклунда с функиией $\eta\left(x, t, u, u_{x}, u_{t}, \ldots\right) \equiv \eta[u]$ такой, что действие его на $F^{0}$ определяется формулой

$$
\left.X_{\eta} F^{0}\right|_{F^{0}=0}=F^{1}
$$

где $F^{0}, F^{1}$ соответствуют уравнению (15). Если $u^{0}$ - решение уравнения (16), то частное решение линеаризованного уравнения задается формулой $u^{1}(x, t)=$ $\eta\left[u^{0}(x, t)\right]$.

ДокАЗАтЕЛЬСтво. Согласно формальной групповой теории, развитой Ибрагимовым [7], каноническому оператору Ли-Беклунда $X_{\eta}$ соответствует формальная однопараметрическая группа преобразований такая, что асимптотическое решение уравнения (15) можно представить в виде ряда

$$
u=u^{0}+\varepsilon \eta\left[u^{0}\right]+\frac{\varepsilon^{2}}{2 !} X_{\eta}(\eta)+\cdots+\frac{\varepsilon^{n}}{n !} X_{\eta}^{n-1}(\eta)+\cdots .
$$

В частности, функция $u^{0}(x, t)+\varepsilon \eta\left[u^{0}(x, t)\right]$ при подстановке в уравнение $(15)$ дает невязку $O\left(\varepsilon^{2}\right)$. Следовательно, $\eta\left[u^{0}(x, t)\right]$ удовлетворяет линеаризованному уравнению. Лемма доказана.

Рассмотрим частный случай уравнения (15):

$$
u_{t}-\frac{1}{2} u_{x}^{2}-u_{x x x}-\varepsilon\left(\frac{1}{3} c_{1} u_{x}^{3}+c_{2} u_{x} u_{x x x}+\frac{1}{2} c_{3} u_{x x}^{2}+c_{4} u_{x x x x x}\right)=0 .
$$


СЛЕДСТВиЕ. Пусть $u^{0}$ - решение потенцированного уравнения КдФ-3: $u_{t}$ $u_{x}^{2} / 2-u_{x x x}=0$, и существует оператор Ли-Беклунда $X_{\eta}$, для которого верна бормула (19) с $F_{0}, F_{1}$ из уравнения (21). Тогда решение линеаризованного уравнения КдФ

$$
u_{t}-u_{x}^{0} u_{x}-u_{x x x}=\frac{1}{3} c_{1}\left(u_{x}^{0}\right)^{3}+c_{2} u_{x}^{0} u_{x x x}^{0}+\frac{1}{2} c_{3}\left(u_{x x}^{0}\right)^{2}+c_{4} u_{x x x x x}^{0}
$$

въчисляется по формуле

$$
u(x, t)=\eta\left[u^{0}(x, t)\right] .
$$

Подобное применение операторов Ли-Беклунда встречается в работах [8]. В частности, там были найдены операторы Ли-Беклунда для уравнения КдФ $u_{t}=6 u u_{x}+$ $u_{x x x}$, и с их помощью построены решения линеаризованного уравнения КдФ. Однако эти результаты не были связаны с задачами из теории поверхностных волн или с уравнением Буссинеска.

\section{5. АСИМПТОТИЧЕСКИЕ РЕШЕНИЯ УРАВНЕНИЯ БУССИНЕСКА}

Теорема. Уравнение Буссинеска (11) имеет асимптотическое решение

$$
u(z, t ; \epsilon)=2 k \operatorname{th} A+2 k \operatorname{th} B+\epsilon u_{1}(z, t ; \epsilon)+\epsilon^{2} u_{2}(z, t ; \epsilon),
$$

где

$$
A=\frac{k(t-z)}{6}+\frac{k^{3} \epsilon t}{54}-\frac{31 k^{5} \epsilon^{2} t}{4860}, \quad B=\frac{k(t+z)}{6}+\frac{k^{3} \epsilon t}{54}-\frac{31 k^{5} \epsilon^{2} t}{4860} .
$$

Функиии $u_{1}, u_{2}$, ограниченные равномерно по $z, t$, вычисляются рекуррентно. Функция (22) удовлетворяет уравнению (11) с точностъю $O\left(\epsilon^{3}\right), \epsilon \rightarrow 0$, равномерно для всех $z, t$.

ДокАЗАтЕЛЬСтво. Известно асимптотическое решение уравнения (11) в главном порядке $u=a(z+t)+b(z-t)$ с произвольными $a, b \in C^{2}$, которое пригодно при $|z|+|t| \ll \epsilon^{-1}$.

Для построения асимптотики, пригодной в бо́льшей области, необходимо учитывать члены порядка $\epsilon$ в уравнении (11). Асимптотическое решение в этом случае берется в виде $u=a(z+t, \tau)+b(z-t, \tau), \tau=\epsilon t$, где функции $a, b$ - решения потенцированного уравнения КдФ. Такое приближение пригодно при $|z|+|t| \ll \epsilon^{-2}$; для более далеких времен надо учитывать члены порядка $\epsilon^{2}$ в уравнении Буссинеска.

Для интегрирования уравнения (11) удобно повернуть систему координат, сделав замену переменных $\xi=t-z, \zeta=t+z$. При этом получается уравнение

$$
\begin{aligned}
u_{\xi \zeta}- & \frac{1}{2} \epsilon\left[u_{\xi} u_{\xi \xi}+u_{\zeta} u_{\zeta \zeta}-\frac{1}{3}\left(u_{\xi} u_{\zeta \zeta}+u_{\zeta} u_{\xi \xi}\right)-\right. \\
& \left.-\frac{2}{3} u_{\xi \zeta}\left(u_{\xi}+u_{\zeta}\right)+u_{\xi \xi \xi \xi}+u_{\zeta \zeta \zeta \zeta}-4 u_{\xi \zeta \zeta \zeta}+6 u_{\xi \xi \zeta \zeta}-4 u_{\xi \xi \xi \zeta}\right]+ \\
& +\epsilon^{2}\left[2\left(u_{\zeta \zeta}-u_{\xi \xi}\right)\left(u_{\zeta \zeta \zeta}-u_{\xi \xi \xi}\right)+\left(u_{\xi}+u_{\zeta}\right)\left(u_{\xi \xi \xi \xi}+u_{\zeta \zeta \zeta \zeta}\right)-\right.
\end{aligned}
$$




$$
\begin{aligned}
& -\frac{1}{6}\left(u_{\xi}^{2} u_{\xi \xi}+u_{\zeta}^{2} u_{\zeta \zeta}-2 u_{\xi} u_{\zeta}\left(u_{\xi \xi}+u_{\zeta \zeta}\right)+u_{\zeta}^{2} u_{\xi \xi}+u_{\xi}^{2} u_{\zeta \zeta}\right)+ \\
& \left.+\frac{6}{5}\left(u_{\xi \xi \xi \xi \xi \xi}+u_{\zeta \zeta \zeta \zeta \zeta \zeta}\right)\right]=O\left(\epsilon^{3}\right), \quad \epsilon \rightarrow 0 .
\end{aligned}
$$

Это уравнение можно преобразовать к асимптотически эквивалентному, исключив смешанные производные $u_{\xi \zeta}$ из младших членов. Так как в силу уравнения (23) $u_{\xi \zeta}=O(\epsilon)$, то из $(23)$ вытекает

$$
\begin{aligned}
u_{\xi \zeta} & =\frac{1}{2} \epsilon\left[u_{\xi} u_{\xi \xi}+u_{\zeta} u_{\zeta \zeta}-\frac{1}{3}\left(u_{\xi} u_{\zeta \zeta}+u_{\zeta} u_{\xi \xi}\right)+u_{\xi \xi \xi \xi}+u_{\zeta \zeta \zeta \zeta}\right]+O\left(\epsilon^{2}\right) \equiv \\
& \equiv \frac{1}{2} \epsilon \Psi+O\left(\epsilon^{2}\right), \quad \epsilon \rightarrow 0 .
\end{aligned}
$$

Подставив $(24)$ в (23) в первом и втором порядках, получаем

$$
\begin{aligned}
u_{\xi \zeta}=\frac{1}{2} & \epsilon \Psi-\frac{1}{6} \varepsilon^{2}\left(u_{\xi}+u_{\zeta}\right) \Psi-\epsilon^{2} \Psi_{\zeta \zeta}+\frac{3}{2} \varepsilon^{2} \Psi_{\xi \zeta}-\varepsilon^{2} \Psi_{\xi \xi}+ \\
& +\varepsilon^{2}\left[2\left(u_{\zeta \zeta}-u_{\xi \xi}\right)\left(u_{\zeta \zeta \zeta}-u_{\xi \xi \xi}\right)+\left(u_{\xi}+u_{\zeta}\right)\left(u_{\xi \xi \xi \xi}+u_{\zeta \zeta \zeta \zeta}\right)-\right. \\
& -\frac{1}{6}\left(u_{\xi}^{2} u_{\xi \xi}+u_{\zeta}^{2} u_{\zeta \zeta}-2 u_{\xi} u_{\zeta}\left(u_{\xi \xi}+u_{\zeta \zeta}\right)+u_{\zeta}^{2} u_{\xi \xi}+u_{\xi}^{2} u_{\zeta \zeta}\right)+ \\
& \left.+\frac{6}{5}\left(u_{\xi \xi \xi \xi \xi \xi}+u_{\zeta \zeta \zeta \zeta \zeta \zeta}\right)\right]+O\left(\epsilon^{3}\right), \quad \epsilon \rightarrow 0 .
\end{aligned}
$$

Для построения асимптотического решения, пригодного при $|\xi|+|\zeta| \ll \epsilon^{-3}$, введем (следуя, например, книге [9]) медленные переменные двух масштабов: $\tau_{1}=$ $\epsilon(\xi+\zeta) / 2, \tau_{2}=\epsilon^{2}(\xi+\zeta) / 2$. Если в искомом решении выделить в явной форме зависимость от дополнительных переменных $u=v\left(\xi, \zeta, \tau_{1}, \tau_{2}, \epsilon\right)$, то исходное уравнение приобретает вид

$$
\begin{aligned}
& v_{\xi \zeta}+\frac{1}{2} \epsilon\left(v_{\tau_{1} \xi}+v_{\tau_{1} \zeta}\right)+\frac{1}{2} \epsilon^{2}\left(v_{\tau_{2} \xi}+v_{\tau_{2} \zeta}\right)+\frac{1}{4} \epsilon^{2} v_{\tau_{1} \tau_{1}}= \\
& =\frac{\epsilon}{2}\left[v_{\xi} v_{\xi \xi}+\epsilon v_{\xi} v_{\tau_{1} \xi}+\frac{1}{2} \epsilon v_{\tau_{1}} v_{\xi \xi}+v_{\zeta} v_{\zeta \zeta}+\right. \\
& +\epsilon v_{\zeta} v_{\tau_{1} \zeta}+\frac{1}{2} \epsilon v_{\tau_{1}} v_{\zeta \zeta}-\frac{1}{3}\left(v_{\xi} v_{\zeta \zeta}+\epsilon v_{\xi} v_{\tau_{1} \zeta}+\frac{\epsilon}{2} v_{\tau_{1}} v_{\zeta \zeta}+\right. \\
& \left.+v_{\zeta} v_{\xi \xi}+\epsilon v_{\zeta} v_{\tau_{1} \xi}+\frac{\epsilon}{2} v_{\tau_{1}} v_{\xi \xi}\right)+v_{\xi \xi \xi \xi}+v_{\zeta \zeta \zeta \zeta}+2 \epsilon\left(v_{\xi \xi \xi \xi \tau_{1}}+\right. \\
& \left.\left.+v_{\zeta \zeta \zeta \zeta \tau_{1}}\right)\right]-\frac{1}{6} \epsilon^{2}\left(v_{\xi}+v_{\zeta}\right) \Psi-\epsilon^{2} \Psi_{\zeta \zeta}+\frac{3}{2} \epsilon^{2} \Psi_{\xi \zeta}-\epsilon^{2} \Psi_{\xi \xi}+ \\
& +\epsilon^{2}\left[2\left(v_{\zeta \zeta}-v_{\xi \xi}\right)\left(v_{\zeta \zeta \zeta}-v_{\xi \xi \xi}\right)+\left(v_{\xi}+v_{\zeta}\right)\left(v_{\xi \xi \xi \xi}+v_{\zeta \zeta \zeta \zeta}\right)-\right. \\
& -\frac{1}{6}\left(v_{\xi}^{2} v_{\xi \xi}+v_{\zeta}^{2} v_{\zeta \zeta}-2 v_{\xi} v_{\zeta}\left(v_{\xi \xi}+v_{\zeta \zeta}\right)+v_{\zeta}^{2} v_{\xi \xi}+v_{\xi}^{2} v_{\zeta \zeta}\right)+ \\
& \left.+\frac{6}{5}\left(v_{\xi \xi \xi \xi \xi \xi}+v_{\zeta \zeta \zeta \zeta \zeta \zeta}\right)\right]+O\left(\epsilon^{3}\right), \quad \epsilon \rightarrow 0 .
\end{aligned}
$$


Асимптотическое решение строим в виде комбинации функций, зависящих от четырех переменных $\left(\xi, \zeta, \tau_{1}, \tau_{2}\right)$ :

$$
u=u^{0}\left(\xi, \zeta, \tau_{1}, \tau_{2}\right)+\epsilon u^{1}\left(\xi, \zeta, \tau_{1}, \tau_{2}\right)+\epsilon^{2} u^{2}\left(\xi, \zeta, \tau_{1}, \tau_{2}\right)+O\left(\epsilon^{3}\right), \quad \epsilon \rightarrow 0 .
$$

Как обычно в таком подходе, зависимость от медленных переменных $\tau_{1}, \tau_{2}$ используется для исключения секулярных членов (слагаемых, неограниченно растущих по своим переменным) в поправках $u^{1}, u^{2}$.

Подставив решение вида (27) в уравнение (26) и приравняв выражения при одинаковых степенях $\epsilon$, получаем последовательность уравнений для $v^{0}, v^{1}, v^{2}$. Зависимость от быстрых переменных $\xi, \zeta$ определяется интегрированием линейного волнового уравнения. Поскольку в главном члене получается однородное уравнение $v_{\xi \zeta}^{0}=0$, то его общее решение выражается через пару произвольных функций, каждая из которых зависит от трех переменных:

$$
v^{0}=a\left(\xi, \tau_{1}, \tau_{2}\right)+b\left(\zeta, \tau_{1}, \tau_{2}\right)
$$

В следующем порядке интегрируется неоднородное уравнение. Для первой поправки получается выражение

$$
v^{1}=\zeta\left(\frac{1}{2} a_{\xi}^{2}+a_{\xi \xi \xi}-a_{\tau_{1}}\right)+\xi\left(\frac{1}{2} b_{\zeta}^{2}+b_{\zeta \zeta \zeta}-b_{\tau_{1}}\right)-\frac{1}{6}\left(a b_{\zeta}+b a_{\xi}\right)+a_{1}+b_{1} .
$$

Здесь функции $a_{1}, b_{1}$ соответствуют общему решению однородного уравнения $u_{\xi \zeta}=0$, так что $a_{1}$ зависит от $\xi, \tau_{1}, \tau_{2}$, а $b_{1}$ зависит от $\zeta, \tau_{1}, \tau_{2}$. В такой форме поправка $v^{1}$ содержит секулярные слагаемые - члены с множителями $\xi$ и $\zeta$. Требование исключения таких слагаемых приводит к хорошо известным уравнениям [3]:

$$
a_{\tau_{1}}=\frac{1}{2} a_{\xi}^{2}+a_{\xi \xi \xi} \quad b_{\tau_{1}}=\frac{1}{2} b_{\zeta}^{2}+b_{\zeta \zeta \zeta}
$$

которые представляют собой два потенцированных уравнения КдФ-3 для функций $a$ и $b$. При таких условиях для поправки $v_{1}$ получается выражение

$$
v^{1}=-\frac{1}{6}\left(a b_{\zeta}+b a_{\xi}\right)+a_{1}+b_{1} .
$$

Аналогично исключение секулярных слагаемых из второй поправки приводит к уравнениям для $a_{1}$ и $b_{1}$ :

$$
\begin{aligned}
a_{1 \tau_{1}}-a_{1 \xi} a_{\xi}-a_{1 \xi \xi \xi} & =\frac{5}{3} a_{\xi} a_{\xi \xi \xi}+\frac{7}{12} a_{\xi \xi}^{2}+\frac{19}{10} a_{\xi \xi \xi \xi \xi}-a_{\tau_{2}}, \\
b_{1 \tau_{1}}-b_{1 \zeta} b_{\zeta}-b_{1 \zeta \zeta \zeta} & =\frac{5}{3} b_{\zeta} b_{\zeta \zeta \zeta}+\frac{7}{12} b_{\zeta \zeta}^{2}+\frac{19}{10} b_{\zeta \zeta \zeta \zeta \zeta}-b_{\tau_{2}} .
\end{aligned}
$$

Эти уравнения для $a_{1}$ и $b_{1}$ представляют собой линеаризацию уравнения КдФ-3 на функциях $a$ и $b$. При их выполнении вторая поправка (без секулярных членов) 
имеет вид

$$
\begin{aligned}
v^{2}=\frac{1}{12} & \left(\frac{1}{2} a_{\xi}^{2} b+\frac{1}{2} b_{\zeta}^{2} a\right)+\frac{1}{12}\left(b_{\zeta} \int \frac{1}{2} a_{\xi}^{2} d \xi+a_{\xi} \int \frac{1}{2} b_{\zeta}^{2} d \zeta\right)+ \\
& +\frac{1}{36}\left(\frac{1}{2} b_{\zeta \zeta} a^{2}+\frac{1}{2} a_{\xi \xi} b^{2}+a_{\xi} \int b b_{\zeta \zeta} d \zeta+b_{\zeta} \int a a_{\xi \xi} d \xi\right)+ \\
& +\frac{13}{12}\left(a_{\xi \xi \xi} b+b_{\zeta \zeta \zeta} a\right)-\frac{23}{12}\left(a_{\xi} b_{\zeta \zeta}+b_{\zeta} a_{\xi \xi}\right)-\frac{1}{6}\left(a b_{1 \zeta}+b a_{1 \xi}+b_{\zeta} a_{1}+a_{\xi} b_{1}\right)
\end{aligned}
$$

Заметим, что вторая поправка $v^{2}$ не содержит секулярных слагаемых в силу условий (30), (31).

Таким образом, построение асимптотических решений сводится к решению уравнений (30), (31) в классе ограниченных функций. По своей постановке такая задача похожа на задачи, возникающие в теории возмущения солитонов [4]. Это сходство подчеркивается еще и тем, что здесь в качестве главного члена асимптотики выбирается аналог солитонного решения (14).

Для получения ограниченных решений $a_{1}, b_{1}$ линеаризованных уравнений КдФ-3 используем зависимость главных членов $a, b$ от второго медленного времени $\tau_{2}$. Существуют разные способы установления этой зависимости (см., например, работу [10]). Здесь указан один из таких способов, по нашему мнению, наиболее эффективный. Он основан на использовании потенцированного уравнения КдФ-5, которое можно выделить из правых частей уравнений (31), как высшей симметрии потенцированного уравнения КдФ-3. При этом системы уравнений для $a$ и для $b$ можно выписывать отдельно. Рассмотрим систему уравнений для $a$ :

$$
a_{\tau_{1}}=\frac{1}{2} a_{\xi}^{2}+a_{\xi \xi \xi}, \quad a_{\tau_{2}}=\left(\frac{1}{6} a_{\xi}^{3}+a_{\xi} a_{\xi \xi \xi}+\frac{1}{2} a_{\xi \xi}^{2}+\frac{3}{5} a_{\xi \xi \xi \xi \xi}\right) c .
$$

Константа $c \in \mathbb{R}$ используется для того, чтобы иметь некоторый произвол в выборе решения $a_{1}$.

Система, составленная из уравнений (33), совместна, так как представлена потенцированным уравнением КдФ-3 и его высшей симметрией - потенцированным уравнением КдФ-5 (КдФ-иерархия). Из множества решений этой системы для построения асимптотики используем решения вида (14), а именно

$$
a=2 k \operatorname{th}\left(\frac{1}{6} k \xi+\frac{1}{54} \tau_{1} k^{3}+\frac{1}{810} \tau_{2} c k^{5}\right),
$$

где $c$ и $k$ - произвольные постоянные. Подходящий выбор константы $c$ будет указан ниже. Формулы для $b$ строятся аналогично. При выбранных таким образом функциях $a$ и $b$ поправка $v^{1}$ будет ограниченной, если будут ограничены функции $a_{1}, b_{1}$.

Заметим, что при указанном в системе (33) выборе функции $a\left(\xi, \tau_{1}, \tau_{2}\right)$ линеаризованное уравнение (31) для $a_{1}$ приобретает вид

$$
\begin{aligned}
a_{1 \tau_{1}} & -a_{1 \xi} a_{\xi}-a_{1 \xi \xi \xi}=\left(\frac{5}{3}-c\right) a_{\xi} a_{\xi \xi \xi}-\left(\frac{1}{2} c\right) \frac{a_{\xi}^{3}}{3}+ \\
+ & \left(\frac{7}{6}-c\right) \frac{a_{\xi \xi}^{2}}{2}+\left(\frac{19}{10}-\frac{3}{5} c\right) a_{\xi \xi \xi \xi \xi} .
\end{aligned}
$$


УтвеРЖДЕНИЕ. Существуют такие значения константы с, при которых решение $a_{1}\left(\xi, \tau_{1}, \tau_{2}\right)$ уравнения (35) оказывается ограниченным равномерно для всех $\xi$, $\tau_{1}, \tau_{2}$.

Для того чтобы найти ограниченные решения уравнения (35), используем лемму. Из следствия ясно, что если существует канонический оператор Ли-Беклунда $X_{\eta}$, то решение линеаризованного уравнения КдФ (35) можно получить по формуле $a_{1}=\eta[a]$. При этом в формуле (21) следует положить $c_{1}=-c / 2, c_{2}=5 / 3-c$, $c_{3}=7 / 6-c, c_{4}=19 / 10-3 c / 5$ с произвольным значением $c \in \mathbb{R}$. Отметим, что требуется найти такие $\eta$, которые дают ограниченное решение $a_{1}$.

Предполагая, что $\eta=\eta\left(\xi, t, u, u_{\xi}, u_{\xi \xi}, u_{\xi \xi \xi}\right) \equiv \eta[u]$ и оператор $X_{\eta}$ удовлетворяет условию (19) для уравнения (21), приходим к следующему уравнению для $\eta$ в частных производных:

$$
\begin{aligned}
\eta_{t}+ & \eta_{u} u_{t}+\eta_{u_{\xi}} u_{t \xi}+\eta_{u_{\xi \xi}} u_{t \xi \xi}+\eta_{u_{\xi \xi \xi}} u_{t \xi \xi \xi}-u_{\xi}\left(\eta_{\xi}+\eta_{u} u_{\xi}+\eta_{u_{\xi}} u_{\xi \xi}+\right. \\
& \left.+\eta_{u_{\xi \xi}} u_{\xi \xi \xi}+\eta_{u_{\xi \xi \xi}} u_{\xi \xi \xi \xi}\right)-D_{\xi}^{3}(\eta)=c_{1} u_{\xi}^{3}+c_{2} u_{\xi} u_{\xi \xi \xi}+\frac{1}{2} c_{3} u_{\xi \xi}^{2}+c_{4} u_{\xi \xi \xi \xi \xi}
\end{aligned}
$$

Подставим в уравнение (36) выражения для $u_{t}: u_{t}=u_{\xi}^{2} / 2+u_{\xi \xi \xi}$, и всех его производных по $\xi$, т.е. $u_{t \xi}, u_{t \xi \xi}, u_{t \xi \xi \xi}$. Получим уравнение в частных производных, которое имеет бесконечно много решений, что дает произвол в выборе $a_{1}, b_{1}$. Найти все решения уравнения (36) довольно сложно, но можно указать подходящие частные решения. Например, при $c=-31 / 6$ получается

$$
\eta[u]=-\frac{5}{3} \xi\left(u_{\xi \xi \xi}+\frac{1}{2} u_{\xi}^{2}\right)-\frac{1}{18} u u_{\xi}-2 u_{\xi \xi} .
$$

Если здесь положить $u=a$, где $a$ задается формулой (34), то согласно лемме получается решение линеаризованного уравнения $a_{1}\left(\xi, \tau_{1}, \tau_{2}\right)=\eta[a]$. Заметим, что при указанном в формуле (37) выборе $\eta$ полученное решение $a_{1}$ ограничено. Построенная таким образом функция $a_{1}\left(\xi, \tau_{1}, \tau_{2}\right)$ представляет собой частное решение неоднородного линеаризованного уравнения (35). Все другие решения отличаются от $a_{1}$ на решения однородного уравнения, вопрос об ограниченности которых был исследован ранее [10]. Утверждение доказано. Аналогичное утверждение имеет место для функции $b_{1}\left(\zeta, \tau_{1}, \tau_{2}\right)$.

Таким образом, вычисляется асимптотическое решение в главном порядке:

$$
\begin{aligned}
u(z, t, \epsilon)=2 k & \operatorname{th}\left(\frac{1}{6} k(t-z)+\frac{1}{54} k^{3} \epsilon t-\frac{31}{4860} k^{5} \epsilon^{2} t\right)+ \\
+ & 2 k \operatorname{th}\left(\frac{1}{6} k(t+z)+\frac{1}{54} k^{3} \epsilon t-\frac{31}{4860} k^{5} \epsilon^{2} t\right)+O(\epsilon), \quad \epsilon \rightarrow 0 .
\end{aligned}
$$

С учетом формул для поправок (29), (32) и соотношений $\tau_{1}=\epsilon t, \tau_{2}=\epsilon^{2} t$ получается асимптотическое решение, которое при подстановке в уравнение (11) дает функцию порядка $O\left(\epsilon^{3}\right), \epsilon \rightarrow 0$, равномерно для любых $t, z$. Этим заканчивается доказательство теоремы. 


\section{6. ЗАКЛЮЧЕНИЕ}

Главным результатом данной работы можно считать систему уравнений вида (33), полученную для главных членов асимптотики $a\left(\xi, \tau_{1}, \tau_{2}\right)$ или $b\left(\zeta, \tau_{1}, \tau_{2}\right)$. В задаче для поверхностных волн здесь впервые обнаружено появление КдФ-иерархии, которая обеспечивает построение асимптотики вплоть до времен $t \approx \epsilon^{-2}$, где приближение КдФ становится непригодным. Роль высших симметрий в похожей задаче впервые была обнаружена в работе [11].

Интерес здесь представляет также подход к исследованию линеаризованных уравнений с применением преобразований Ли-Беклунда. Его можно использовать при построении решений других линеаризованных уравнений.

Благодарности. Автор выражает благодарность Л. А. Калякину за многочисленные обсуждения и интерес к работе, а также Г. И. Бурде за предоставленную информацию о работе [8], О. М. Киселеву и Б. И. Сулейманову за полезные замечания.

Все сложные вычисления выполнены с применением программы Maple.

\section{Список литературы}

[1] Л. В. Овсянников, “Лагранжевы приближения в теории волн”, Нелинейные проблемъ теории поверхностных и внутренних волн, Наука, Новосибирск, 1985, 10-77.

[2] L. Debnath, Nonlinear Water Waves, Academic Press, Boston, MA, 1983.

[3] Н. И. Макаренко, "The second long-wave approximation in the Cauchy-Poisson problem", Динамика сплошной средъ, № 77, Ин-т гидродинамики АН СССР, Новосибирск, 1986, 56-72; W. Craig, Commun. Part. Diff. Equat., 10:8 (1985), 787-1003; T. Kano, T. Nishida, Osaka J. Math., 23:2 (1986), 389-413; Л.В.Овсянников, "К обоснованию теории мелкой воды”, Динамика сплошной среды, № 15, Ин-т гидродинамики АН СССР, Новосибирск, 1973, 104-125.

[4] В. И. Карпман, Е. М. Маслов, ЖКЭТФ, 73:2 (1977), 281-291; Е. М. Маслов, ТМФ, 42:3 (1980), 362-373; В.П. Маслов, Г. А. Омельянов, УМН, 36:3 (1981), 63-126; Сиб. матем. журн., 24:5 (1983), 172-182; А. Ньюэлл, "Обратное преобразование рассеяния", Солитонь, ред. Р. Буллаф, Ф. Кодри, Мир, М., 1983, 193-269; Л. А. Калякин, ТМФ, 92:1 (1992), 62-76.

[5] V. A. Baikov, S. A. Kordyukova, Quaest. Math., 26 (2003), 1-14; S. A. Kordyukova, Nonlin. Dyn., 46:1-2 (2006), 73-85.

[6] С. Ю. Доброхотов, Докл. АН СССР, 292 (1987), 63-67.

[7] N.H. Ibragimov, R. L. Anderson, "Lie-Bäcklund symmetries: Representation by formal power series", CRC Handbook of Lie Group Analysis of Differential Equation, Vol. 3. New Trends in Theoretical Developments and Computational Methods, ed. N. H. Ibragimov, CRC Press, Boca Raton, 1996, 3-29.

[8] J. Kodama, Phys. Lett. A, 112:5 (1985), 193-196; G. I. Burde, Nonlinearity, 18 (2005), 1443-1461.

[9] А. Найфэ, Методы возмущений, Мир, М., 1976.

[10] Л. А. Калякин, Матем. заметки, 50:5 (1991), 32-42.

[11] A. Degasperis, S. V. Manakov, P. M. Santini, Physica D, 100 (1997), 187-211. 